\title{
The Anatomical Variability of the Human
} Cerebellar Nuclei

\begin{tabular}{|l|l|l|}
\hline J.C. & & Yap \\
\hline J.D.W. & Tomlinson \\
\hline E.S. & & Watkins \\
\hline
\end{tabular}

Neurosurgical Unit, The London Hospital, London

Abstract

Published studies of the anatomy and variability of the dentate nucleus in relation to landmarks of the fourth ventricle are few in number and limited in extent. To determine the variability of this structure, measurements have been made in sufficient numbers of specimens to be statistically significant. Finite radiological relationships were established and the variability of possible radiological reference planes estimated from 48 stereotaxic ventriculograms. Thirty cerebellar hemispheres obtained without special methods of fixation were sectioned at 1-mm intervals and the nuclear variability measured from the most reliable reference plane. A high degree of variability was found which may explain the varying therapeutic effect of dentatotomy and the dissimilar physiological effects reported. To further eliminate anatomical variability due to distortion during fixation, a further 30 cerebellar specimens obtained by a partial decapitation technique with fixation in situ in the skull have been sectioned after stereotaxic marking in a sarcophagus [Andrew and Watkins, 1969] and the dentate and roof nuclear measurements and variability established.

\section{Reference}

